\title{
ON THE LIPSCHITZ CONTINUITY OF THE SOLUTIONS OF A CLASS OF ELLIPTIC FREE BOUNDARY PROBLEMS
}

\section{A. LYAGHFOURI}

Received February 23, 2007 and, in revised form, March 7, 2008

\begin{abstract}
In this article we prove local interior and boundary Lipschitz continuity of the solutions of a general class of elliptic free boundary problems in divergence form.
\end{abstract}

\section{INTRODUCTION}

The purpose of this paper is to study the regularity of the solutions of the following elliptic free boundary problem in divergence form

$$
\left(P_{0}\right)\left\{\begin{array}{l}
\text { Find }(u, \chi) \text { such that: } \\
(i) u \geq 0, \quad 0 \leq \chi \leq 1, \quad u(1-\chi)=0 \quad \text { a.e. in } \Omega \\
(i i) u=\varphi \quad \text { on } \Gamma_{1} \\
(i i i)(a \nabla u+u B+\chi H) \cdot \eta=0 \quad \text { on } \partial \Omega \backslash \Gamma_{1} \\
(i v)-\operatorname{div}(a \nabla u+u B+\chi H)=\chi c+C . \nabla u+d u+f
\end{array}\right.
$$

where $\Omega$ is a bounded domain of $\mathbb{R}^{n}$ with $\partial \Omega=\Gamma_{1} \cup \Gamma_{2}, a(x)=\left(a_{i j}(x)\right)$ is a $n$-by- $n$ matrix, $x=\left(x_{1}, \ldots, x_{n}\right), B(x)=\left(b_{1}(x), \ldots, b_{n}(x)\right), C(x)=$

2000 Mathematics Subject Classification. Primary: 35B65, 35R35.

Key words and phrases. Free boundary, Lipschitz continuity.

ISSN 1425-6908（C) Heldermann Verlag. 
$\left(c_{1}(x), \ldots, c_{n}(x)\right), H(x)=\left(h_{1}(x), \ldots, h_{n}(x)\right)$ are vector functions, $c(x)$, $d(x)$ and $f(x)$ are real valued functions. $\eta$ is the outward unit normal vector to $\partial \Omega$.

Note that for $B(x)=C(x)=0, d(x)=c(x)=f(x)=0$ and $H(x)=$ $a(x) e_{n}$, with $e_{n}=(0, \ldots, 0,1),\left(P_{0}\right)$ corresponds to the dam problem with Dirichlet boundary conditions (see [1], [4]).

When $B(x)=C(x)=H(x)=0$ and $d(x)=f(x)=0$, we have the obstacle problem.

When $n=2, a(x)=h^{3}(x) I_{2}, B(x)=C(x)=0, d(x)=c(x)=f(x)=0$ and $H(x)=h(x) e_{2}$, where $I_{2}$ is the 2-by-2 identity matrix and $h(x)$ is a scalar function, we have the lubrication problem.

When $n=2, a(x)=k(x) I_{2}, B(x)=C(x)=0, d(x)=c(x)=f(x)=0$ and $H(x)=h(x) e_{1}$, with $e_{1}=(1,0), k(x)$ and $h(x)$ are scalar functions, we have the aluminium electrolysis problem.

Given the jump condition along the free boundary $(\partial[u>0]) \cap \Omega$, the optimal expected regularity for a solution $u$ is Lipschitz continuity. This regularity result was proved in [1] for the dam problem and was extended in [2]. The objective of this paper is to consider a more general class of problems and also to establish a similar regularity up to $\Gamma_{1}$.

The main idea of the proof of the interior regularity is the comparison of $u$ with a function of type $v(x)=k\left(e^{-\mu \rho^{2}}-e^{-\mu(r+\delta)^{2}}\right)$ in order to derive an estimate of the form $u\left(x_{0}\right) \leq C r$ whenever $B\left(x_{0}, r\right)$ is a maximal open ball satisfying $B\left(x_{0}, r\right) \subset[u>0], \overline{B\left(x_{0}, r\right)} \subset \Omega$. For the regularity up to the boundary, we first reduce the problem to a flat boundary. The main idea then is the estimate $u(x) \leq C\left|x-x_{0}\right|$ in the half ball $B^{+}\left(x_{0}, R\right)$, where $x_{0} \in \Gamma_{1}$. This is obtained by comparing $u$ to the test function $\psi\left(\mid x-x_{0}+\right.$ $\left.R e_{n} \mid-R\right)$, where $\psi(t)=-\beta / \alpha t+\left(\beta / \alpha^{2}\right) e^{2 \alpha R}\left(1-e^{-\alpha t}\right)$, and $\alpha, \beta$ are some constants.

\section{Statement of the Problem}

Throughout this paper we assume that $\Omega$ is a Lipschitz bounded domain of $\mathbb{R}^{n}$ and that

$$
\begin{array}{ll}
\sum\left|a_{i j}(x)\right|^{2} \leq M^{2}, & \text { for a.e. } x \in \Omega,(1.1) \\
a(x) \xi . \xi \geq \lambda|\xi|^{2} \quad \forall \xi \in \mathbb{R}^{2}, & \text { for a.e. } x \in \Omega,(1.2) \\
\lambda^{-2} \sum_{1}\left(\left|b_{i}(x)\right|^{2}+\left|c_{i}(x)\right|^{2}\right)+\lambda^{-1}|d(x)| \leq \nu^{2} & \\
d+\operatorname{div}(B) \leq 0 & \text { in } \mathcal{D}^{\prime}(\Omega) \\
H \in \mathbb{L}^{2}(\Omega) &
\end{array}
$$$$
\text { for a.e. } x \in \Omega,(1.2)
$$ 


$$
\begin{array}{ll}
c \in L^{2}(\Omega) & \\
f \in L^{2}(\Omega), & f \geq 0 \text { a.e. in } \Omega \\
\varphi \in C^{0,1}(\bar{\Omega}), & \varphi \geq 0 \text { a.e. in } \Omega
\end{array}
$$

where $\lambda, \nu$ and $M$ are positive constants. Note that assumption (1.4) and the nonnegativity of $f$ and $\varphi$ are needed in order to get nonnegative solutions.

We shall denote by $B(x, r)$ an open ball of center $x$ and radius $r$. By $L$ we denote the linear operator defined by

$$
L u=\operatorname{div}(a(x) \nabla u+u B(x))+C(x) \nabla u+d(x) u .
$$

We consider then the following weak formulation of problem $\left(P_{0}\right)$

$$
(P)\left\{\begin{array}{l}
\text { Find }(u, \chi) \in H^{1}(\Omega) \times L^{\infty}(\Omega) \text { such that: } \\
(i) \quad u \geq 0, \quad 0 \leq \chi \leq 1, \quad u(1-\chi)=0 \text { a.e. in } \Omega \\
(i i) \quad u=\varphi \quad \text { on } \Gamma_{1} \\
(i i i) \int_{\Omega}(a(x) \nabla u+u B(x)+\chi H(x)) \cdot \nabla \xi \\
\quad-(\chi c(x)+C(x) . \nabla u+d(x) u) \xi d x=\int_{\Omega} f \xi d x \\
\forall \xi \in H^{1}(\Omega), \quad \xi=0 \text { on } \Gamma_{1} .
\end{array}\right.
$$

First we have the following proposition.

Proposition 1.1. There exists a solution $(u, \chi)$ of $(P)$ such that $u \leq$ $\max _{\bar{\Gamma}_{1}} \varphi=U$.

Proof. For the existence of a solution of $(P)$, we consider for $\varepsilon>0$ the approximated problem

$$
\left(P_{\varepsilon}\right)\left\{\begin{array}{l}
\text { Find } u_{\varepsilon} \in H^{1}(\Omega) \text { such that: } \\
(i) \quad u_{\varepsilon}=\varphi \text { on } \Gamma_{1} \\
(i i) \quad \int_{\Omega}\left(a(x) \nabla u_{\varepsilon}+u_{\varepsilon} B(x)+h_{\varepsilon}\left(u_{\varepsilon}\right) H(x)\right) \cdot \nabla \xi \\
\quad-\left(h_{\varepsilon}\left(u_{\varepsilon}\right) c(x)+C(x) \cdot \nabla u_{\varepsilon}+d(x) u_{\varepsilon}\right) \xi d x=\int_{\Omega} f \xi d x \\
\quad \forall \xi \in H^{1}(\Omega), \quad \xi=0 \text { on } \Gamma_{1}
\end{array}\right.
$$

where $h_{\varepsilon}(t)=\min \left(t^{+} / \varepsilon, 1\right)$. 
To prove the existence of a solution of $\left(P_{\varepsilon}\right)$, we consider for each $v \in$ $L^{2}(\Omega)$, the following problem

$$
\left(P_{\varepsilon}^{v}\right)\left\{\begin{array}{l}
\text { Find } u_{\varepsilon} \in H^{1}(\Omega) \text { such that: } \\
(i) u_{\varepsilon}=\varphi \quad \text { on } \Gamma_{1} \\
(i i) \int_{\Omega}\left(a(x) \nabla u_{\varepsilon}+u_{\varepsilon} B(x)+h_{\varepsilon}(v) H(x)\right) \cdot \nabla \xi \\
\quad-\left(h_{\varepsilon}(v) c(x)+C(x) \cdot \nabla u_{\varepsilon}+d(x) u_{\varepsilon}\right) \xi d x=\int_{\Omega} f \xi d x \\
\forall \xi \in H^{1}(\Omega), \quad \xi=0 \text { on } \Gamma_{1} .
\end{array}\right.
$$

Under the assumptions (1.1)-(1.8), there exists at least one solution $u_{\varepsilon}$ to $\left(P_{\varepsilon}^{v}\right)$ (see $[3$, p. 215]). Then we apply the Schauder fixed point theorem to get a solution of $\left(P_{\varepsilon}\right)$. Using $u_{\varepsilon}^{-}$as a test function and arguing as in [3, Theorem 8.1, p. 179], we obtain $u_{\varepsilon} \geq 0$ a.e. in $\Omega$. Finally we establish an a priori estimate of $\left|u_{\varepsilon}\right|_{1,2}$ and use the fact that $0 \leq h_{\varepsilon}\left(u_{\varepsilon}\right) \leq 1$ a.e in $\Omega$ to pass to the limit and get a solution of the problem $(P)$.

To establish the estimate one can adapt the proof of Theorem 8.1 in linebreak [3, p. 179].

\section{Remark 1.1.}

i) If for $\zeta \in \mathcal{D}(\Omega)$, one takes $\pm \zeta$ as test functions in $(P)$ (iii), one gets $L u=-f-\chi c(x)-\operatorname{div}(\chi H)$ in $\mathcal{D}^{\prime}(\Omega)$. Then if $c, f \in L_{\mathrm{loc}}^{p}(\Omega)$ and $H \in \mathbb{L}_{\text {loc }}^{2 p}(\Omega)$ with $p>n / 2$, and if we take into account (1.1)-(1.8), we obtain (see [3, Theorem 8.29, p. 205]) that $u \in C_{\text {loc }}^{0, \alpha}\left(\Omega \cup \Gamma_{1}\right)$ for some $\alpha \in(0,1)$. In particular the set $[u>0]$ is open.

ii) Due to $(P)(i)$, we have $L u=-f-c(x)-\operatorname{div}(H)$ in $\mathcal{D}^{\prime}([u>0])$. So if $a, b_{i} \in C_{\mathrm{loc}}^{0, \alpha}(\Omega)(0<\alpha<1), c_{i}, d \in L_{\mathrm{loc}}^{\infty}(\Omega), c, f, \operatorname{div}(H) \in L_{\mathrm{loc}}^{p}(\Omega)$ with $p>n /(1-\alpha)$, we obtain (see $\left[3\right.$, p. 212]) that $u \in C_{\mathrm{loc}}^{1, \alpha}([u>0])$.

\section{Interior Lipschitz CONTINUity}

In this section we assume that

$$
\begin{aligned}
& a, b_{i} \in C_{\mathrm{loc}}^{0, \alpha}(\Omega), \quad c_{i}, d \in L_{\mathrm{loc}}^{\infty}(\Omega) \quad i=1, \ldots, n, \alpha \in(0,1) \\
& \forall s>0, \forall x_{0} \in \Omega, \forall y \in B\left(x_{0}, s\right) \subset \Omega: \operatorname{div}(a(x)(x-y)) \leq c_{0}(s) \\
& \text { in } \mathcal{D}^{\prime}\left(B\left(x_{0}, s\right)\right) \\
& c, f, \operatorname{div}(H) \in L_{\mathrm{loc}}^{p}(\Omega), \quad p>n /(1-\alpha) \\
& |H(x)| \leq c_{0}(s) \quad \text { a.e. in } B\left(x_{0}, s\right) \\
& -c^{-}+\operatorname{div}(H) \geq-c_{0}(s) \quad \text { in } \mathcal{D}^{\prime}\left(B\left(x_{0}, s\right)\right) \\
& d+\operatorname{div}(B) \geq-c_{0}(s) \quad \text { in } \mathcal{D}^{\prime}\left(B\left(x_{0}, s\right)\right)
\end{aligned}
$$


where $c_{0}$ is a positive constant depending only on $s$. Without loss of generality, we can assume that $\lambda<1 / 2$.

The main result of this section is the following theorem.

Theorem 2.1. Let $(u, \chi)$ be a solution of $(P)$. Then $u \in C_{\mathrm{loc}}^{0,1}(\Omega)$.

To prove Theorem 2.1, we need three lemmas.

Lemma 2.1. Let $x_{0}=\left(x_{01}, \ldots, x_{0 n}\right)$ and $r, s>0$ such that $r \leq r_{0}=$ $(\sqrt{7}-2) /(8 \nu), B\left(x_{0}, r\right) \subset[u>0], \overline{B\left(x_{0}, r\right)} \subset B\left(x_{0}, s\right) \subset \Omega$ and $\partial B\left(x_{0}, r\right) \cap$ $\partial[u>0] \neq \emptyset$. Then we have for some positive constant $C$ depending only on $\lambda, \nu, U$ and $s$, but not on $r$

$$
\min _{B_{r / 2}\left(x_{0}\right)} u \leq C r .
$$

Proof. Let $m=\min \frac{}{B\left(x_{0}, r / 2\right)} u$ and $\delta \in(0, r)$ such that $B\left(x_{0}, r+\delta\right) \subset$ $B\left(x_{0}, s\right)$, and let $v$ be defined by

$$
v(x)=k\left(e^{-\mu \rho^{2}}-e^{-\mu(r+\delta)^{2}}\right)
$$

where

$$
\begin{aligned}
\rho^{2} & =\sum_{i=1}^{i=n}\left(x_{i}-x_{0 i}\right)^{2}, \quad k=\frac{m}{\left(e^{-\mu r^{2} / 4}-e^{-\mu(r+\delta)^{2}}\right)}, \quad \mu=\frac{\kappa}{r^{2}} \quad \text { and } \\
\kappa & =4 \nu s+\frac{2 c_{0}+1}{\lambda} .
\end{aligned}
$$

Then one has

$$
\begin{cases}L v \geq-\chi c-f-\operatorname{div}(H) & \text { in } D=B\left(x_{0}, r+\delta\right) \backslash \overline{B\left(x_{0}, r / 2\right)} \\ v=m & \text { on } \partial B\left(x_{0}, r / 2\right) \\ v=0 & \text { on } \partial B\left(x_{0}, r+\delta\right) \\ |\nabla v|=2 k \mu \rho e^{-\mu \rho^{2}} & \text { decreases with respect to } \rho .\end{cases}
$$

Indeed we have $\nabla v=-2 \mu k e^{-\mu \rho^{2}}\left(x-x_{0}\right)$ and therefore we get

$$
\begin{aligned}
\frac{d}{d \rho}|\nabla v|= & 2 k \mu e^{-\mu \rho^{2}}\left(1-2 \kappa \frac{\rho^{2}}{r^{2}}\right) \leq 2 k \mu e^{-\mu \rho^{2}}(1-\kappa / 2) \leq 0 \\
& \text { since } \kappa>1 / \lambda>2 \\
L v= & 4 \mu^{2} k e^{-\mu \rho^{2}} a(x)\left(x-x_{0}\right) \cdot\left(x-x_{0}\right)-2 \mu k e^{-\mu \rho^{2}} \operatorname{div}\left(a(x)\left(x-x_{0}\right)\right) \\
& -2 \mu k e^{-\mu \rho^{2}}(B(x)+C(x))\left(x-x_{0}\right)+(d(x)+\operatorname{div}(B)) v \\
\geq & 4 \mu^{2} \lambda\left(r^{2} / 4\right) k e^{-\mu \rho^{2}}-2 \mu k c_{0} e^{-\mu \rho^{2}}-4 \mu s \lambda \nu k e^{-\mu \rho^{2}}-c_{0} U \\
\geq & \mu k\left[\mu \lambda r^{2}-4 \lambda \nu s-2 c_{0}\right] e^{-\mu \rho^{2}}-c_{0} U
\end{aligned}
$$




$$
\begin{aligned}
& =\kappa \frac{m}{r^{2}}\left[\kappa \lambda-4 \lambda \nu s-2 c_{0}\right] \frac{e^{-\mu \rho^{2}}}{e^{-\mu r^{2} / 4}-e^{-\mu(r+\delta)^{2}}}-c_{0} U \\
& =\kappa \frac{m}{r^{2}} \frac{e^{-\mu \rho^{2}}}{e^{-\mu r^{2} / 4}-e^{-\mu(r+\delta)^{2}}}-c_{0} U=\kappa \frac{m}{r^{2}} \frac{e^{-\mu \rho^{2}} e^{\mu(r+\delta)^{2}}}{e^{\mu\left((r+\delta)^{2}-r^{2} / 4\right)}-1}-c_{0} U \\
& \geq \kappa \frac{m}{r^{2}} \frac{1}{e^{\mu\left((r+\delta)^{2}-r^{2} / 4\right)}-1}-c_{0} U \\
& \geq \kappa \frac{m}{r^{2}} \frac{1}{e^{\mu\left(4 r^{2}\right)}-1}-c_{0} U \quad \text { since } 0<\delta<r \\
& =\kappa \frac{m}{r^{2}} \frac{1}{e^{4 \kappa}-1}-c_{0} U .
\end{aligned}
$$

Using (1.7) and (2.5), we get from (2.7)

$$
\begin{aligned}
L v+f+\chi c+\operatorname{div}(H) & \geq \kappa \frac{m}{r^{2}} \frac{1}{e^{4 \kappa}-1}-c_{0} U-\chi c^{-}+\operatorname{div}(H) \\
& \geq \kappa \frac{m}{r^{2}} \frac{1}{e^{4 \kappa}-1}-c_{0} U-c_{0} .
\end{aligned}
$$

Now if

$$
m \leq \frac{c_{0}(1+U)\left(e^{4 \kappa}-1\right)}{\kappa} r^{2}, \quad \text { then } \quad m \leq \frac{s c_{0}(1+U)\left(e^{4 \kappa}-1\right)}{\kappa} r
$$

and the lemma is proved. Assume that

$$
m>\frac{c_{0}(1+U)\left(e^{4 \kappa}-1\right)}{\kappa} r^{2} .
$$

Then we obtain from $(2.8)$

$$
L v+f+\chi c+\operatorname{div}(H) \geq 0 \quad \text { in } D .
$$

Since $v \leq u$ on $\partial D, \zeta=(v-u)^{+} \in H_{0}^{1}(D)$, and $\pm \zeta$ - after being extended by zero outside $D$ - are test functions for $(P)$. So we have

$$
\begin{aligned}
& \int_{D}(a(x) \nabla u+u B(x)+\chi H(x)) \cdot \nabla(v-u)^{+} \\
& -(\chi c(x)+C(x) \cdot \nabla u+d(x) u)(v-u)^{+} d x=\int_{D} f(v-u)^{+} d x .
\end{aligned}
$$

By (2.9) we have

$$
\begin{aligned}
& \int_{D}(a(x) \nabla v+v B(x)+H(x)) \cdot \nabla(v-u)^{+} \\
& -(\chi c(x)+C(x) \cdot \nabla v+d(x) v)(v-u)^{+} d x \leq \int_{D} f(v-u)^{+} d x .
\end{aligned}
$$

Subtracting (2.10) from (2.11), we get

$$
\int_{D} a(x) \nabla(v-u) \cdot \nabla(v-u)^{+} d x \leq \int_{D}(v-u)^{+}(C(x)-B(x)) \cdot \nabla(v-u)^{+}
$$




$$
\begin{aligned}
& +\int_{D}(\chi-1) H(x) \cdot \nabla(v-u)^{+} \\
& +\int_{D} d(x)(v-u)^{+2} d x
\end{aligned}
$$

which can be written by (1.2)-(1.3) and by taking into account that $\chi=1$ a.e. in $[u>0]$

$$
\begin{aligned}
\int_{D} \lambda\left|\nabla(v-u)^{+}\right|^{2} d x \leq & 2 \lambda \nu \int_{D}(v-u)^{+}\left|\nabla(v-u)^{+}\right| d x+\int_{D} \lambda \nu^{2}(v-u)^{+2} d x \\
& +\int_{D \cap[u=0]}(\chi-1) H(x) . \nabla v d x
\end{aligned}
$$

Note that we have by Cauchy-Schwarz inequality

$$
\begin{aligned}
& \int_{D}(v-u)^{+}\left|\nabla(v-u)^{+}\right| d x \\
& \leq\left(\int_{D}(v-u)^{+2} d x\right)^{1 / 2} \cdot\left(\int_{D}\left|\nabla(v-u)^{+}\right|^{2} d x\right)^{1 / 2} .
\end{aligned}
$$

Now using Poincaré's inequality and majoring the constant, we obtain

$$
\int_{D}(v-u)^{+2} d x \leq 16 r^{2} \int_{D}\left|\nabla(v-u)^{+}\right|^{2} d x .
$$

Therefore we deduce from (2.12)-(2.14) that

$$
\begin{aligned}
\int_{D} \lambda\left|\nabla(v-u)^{+}\right|^{2} d x \leq & 8 \lambda \nu r \int_{D}\left|\nabla(v-u)^{+}\right|^{2} d x+16 \lambda \nu^{2} r^{2} \int_{D}\left|\nabla(v-u)^{+}\right|^{2} d x \\
& +\int_{D \cap[u=0]} c_{0}|\nabla v| d x
\end{aligned}
$$

which can be written

$$
\lambda\left(1-8 \nu r-16 \nu^{2} r^{2}\right) \int_{D}\left|\nabla(v-u)^{+}\right|^{2} d x \leq \int_{D \cap[u=0]} c_{0}|\nabla v| d x .
$$

This leads for $r \leq r_{0}=(\sqrt{7}-2) /(8 \nu)$ to

or

$$
\frac{\lambda}{4} \int_{D}\left|\nabla(v-u)^{+}\right|^{2} d x \leq \int_{D \cap[u=0]} c_{0}|\nabla v| d x
$$

$$
\int_{D \cap[u>0]}\left|\nabla(v-u)^{+}\right|^{2} d x \leq \int_{D \cap[u=0]}|\nabla v|\left(\frac{4 c_{0}}{\lambda}-|\nabla v|\right) d x .
$$

Now we claim that

$$
\int_{D \cap[u>0]}\left|\nabla(v-u)^{+}\right|^{2} d x>0 .
$$


Indeed assume that

$$
\int_{D \cap[u>0]}\left|\nabla(v-u)^{+}\right|^{2} d x=0 .
$$

In particular we have

$$
\int_{B\left(x_{0}, r\right) \backslash \overline{B\left(x_{0}, r / 2\right)}}\left|\nabla(v-u)^{+}\right|^{2} d x=0
$$

and then $\nabla(v-u)^{+}=0$ in $B\left(x_{0}, r\right) \backslash \overline{B\left(x_{0}, r / 2\right)}$. Since $v \leq u$ on $\partial B\left(x_{0}, r / 2\right)$, we get $v \leq u$ in $B\left(x_{0}, r\right) \backslash \overline{B_{r / 2}}\left(x_{0}\right)$. This leads to a contradiction with $\partial B\left(x_{0}, r\right) \cap \partial[u>0] \neq \emptyset$ and $v>0$ in $D$. Hence (2.16) is true and we deduce from (2.15) that

$$
\int_{D \cap[u=0]}|\nabla v|\left(\frac{4 c_{0}}{\lambda}-|\nabla v|\right) d x>0 .
$$

Since $|\nabla v|$ is non-increasing with respect to $\rho$, we infer from $(2.17)$ that

$$
|\nabla v|_{\left.\right|_{\partial B\left(x_{0}, r+\delta\right)}}=2 k \mu(r+\delta) e^{-\mu(r+\delta)^{2}}<\frac{4 c_{0}}{\lambda} .
$$

Letting $\delta \rightarrow 0$, we get

$$
m \leq \frac{2 c_{0}}{\kappa \lambda}\left(e^{3 \kappa / 4}-1\right) r=C r
$$

Lemma 2.2. Assume that $u>0$ in $B\left(x_{0}, \mu r\right) \subset B\left(x_{0}, s\right) \subset \Omega$. Then

$$
u_{r}(y)=\frac{u\left(x_{0}+r y\right)}{r}
$$

is defined in $B(O, \mu)$, where $B(O, \mu)$ is the open ball of center $O=(0, \ldots, 0)$ and radius $\mu$. Moreover we have

i) $\operatorname{div}\left(a_{r}(y) \nabla u_{r}+u_{r} B_{r}(y)\right)-\left(r C_{r}(y) \nabla u_{r}+r^{2} d_{r}(y) u_{r}\right)=r c_{r}(y)+r f_{r}(y)+$ $\operatorname{div}\left(H_{r}\right)$ in $B_{\mu}$, where $g_{r}(y)=g\left(x_{0}+r y\right)$.

ii) $\max _{B\left(x_{0}, \mu r\right)} u \leq C\left(\min _{B\left(x_{0}, \mu r / 2\right)} u+r\right)$ where $C$ is a positive constant depending only on $\mu, \lambda, \nu, M, U, n, p$ and $s$.

iii) $\sup _{B\left(x_{0}, \mu r / 2\right)}|\nabla u| \leq C\left(\frac{\sup _{B\left(x_{0}, \mu r\right)} u}{r}+1\right)$ where $C$ is a positive constant depending only on $\mu, \lambda, \nu, M, U, n, p$, $\left|a_{i j}\right|_{0, \alpha, B\left(x_{0}, s\right)},\left|b_{i}\right|_{0, \alpha, B\left(x_{0}, s\right)},\left|c_{i}\right|_{0, B\left(x_{0}, s\right)},|d|_{0, B\left(x_{0}, s\right)}$ and $s$. 
Proof. i) Straightforward calculation.

ii) Since $r$ is bounded by $s / \mu$, we can assume that $a_{r}, B_{r}, r C_{r}, r d_{r}$, satisfy the assumptions (1.1)-(1.3) with the constants independent on $r$. Therefore we can apply Theorems 8.17 and 8.18 in [3, p. 194] (Harnack inequality) to the equation in i). We get for a positive constant $C_{1}$ depending only on $\mu$, $\lambda, \nu, M, U, n, p$ and $s$

$$
\max _{B(O, \mu / 2)} u_{r} \leq C_{1}\left(\min _{B(O, \mu / 2)} u_{r}+\left|r\left(c_{r}+f_{r}\right)\right|_{L^{p}\left(B_{\mu}\right)}+\left|H_{r}\right|_{L^{2 p}\left(B_{\mu}\right)}\right) .
$$

Since $p>n /(1-\alpha)>n$ and due to $(2.3)$, we have for some constant $C_{2}(s)$

$$
\begin{aligned}
\left|r\left(c_{r}+f_{r}\right)\right|_{L^{p}\left(B_{\mu}\right)} & =\left(\int_{B_{\mu}} r^{p}|c+f|^{p}\left(x_{0}+r y\right) d y\right)^{1 / p} \\
& =\left(\int_{B\left(x_{0}, \mu r\right)} \frac{r^{p}}{r^{n}}|c+f|^{p}(x) d x\right)^{1 / p} \\
& =r^{1-n / p}|c+f|_{L^{p}\left(B\left(x_{0}, \mu r\right)\right)} \leq C_{2} r^{1-n / p} \\
\left|H_{r}\right|_{L^{2 p}(B(O, \mu))} & =\left(\int_{B(O, \mu)}|H|^{2 p}\left(x_{0}+r y\right) d y\right)^{1 / 2 p} \leq c_{0}(s)|B(O, \mu)|^{1 / 2 p} .
\end{aligned}
$$

Hence we obtain for a constant $C_{3}$ independent of $r$

$$
\max _{B(O, \mu / 2)} u_{r} \leq C_{3}\left(\min _{B(O, \mu / 2)} u_{r}+r^{1-n / p}+1\right) .
$$

Now using the definition of $u_{r}$, one can verify that

$$
\max _{B(O, \mu / 2)} u_{r}=\frac{1}{r} \max _{B\left(x_{0}, \mu r / 2\right)} u \text { and } \min _{B(O, \mu / 2)} u_{r}=\frac{1}{r} \min _{B\left(x_{0}, \mu r / 2\right)} u .
$$

It follows that

$$
\max _{B\left(x_{0}, \mu r / 2\right)} u \leq C_{3}\left(\min _{B\left(x_{0}, \mu r / 2\right)} u+r^{2-n / p}+r\right) .
$$

Finally since $p>n$ and $r$ is bounded, we have $r^{2-n / p} \leq C_{4} r$, which leads finally to

$$
\max _{B\left(x_{0}, \mu r / 2\right)} u \leq C\left(\min _{B\left(x_{0}, \mu r / 2\right)} u+r\right) .
$$

iii) From the equation in i), we know (see [3, p. 212], Corollary 8.36 and the Remark after it) that $u_{r} \in C^{1, \alpha}(B(O, \mu))$ and that

$$
\begin{aligned}
& \left|u_{r}\right|_{1, \alpha, \overline{B(O, \mu / 2)}} \\
& \leq C\left(\left|u_{r}\right|_{0, B_{\mu}}+\left|r c_{r}\right|_{p, B(O, \mu)}+\left|r f_{r}\right|_{p, B(O, \mu)}+\left|\operatorname{div}\left(H_{r}\right)\right|_{p, B(O, \mu)}\right),
\end{aligned}
$$


where $C$ depends only on $\operatorname{dist}\left(\overline{B(O, \mu / 2)}, \partial B_{\mu}\right), \lambda, \quad \nu, \quad M, U, \quad n, \quad p$, $\left|a_{i j}\right|_{0, \alpha, B\left(x_{0}, s\right)},\left|b_{i}\right|_{0, \alpha, B\left(x_{0}, s\right)},\left|c_{i}\right|_{0, B\left(x_{0}, s\right)},|d|_{0, B\left(x_{0}, s\right)}$ and $s$. In particular, we obtain $\sup _{B(O, \mu / 2)}\left|\nabla u_{r}\right| \leq C\left(\sup _{B(O, \mu)} u_{r}+1\right)$ or equivalently

$$
\sup _{B\left(x_{0}, \mu r / 2\right)}|\nabla u| \leq C\left(\frac{\sup _{B\left(x_{0}, \mu r\right)} u}{r}+1\right) .
$$

Lemma 2.3. Under the assumptions of Lemma 2.1, we have for a constant $C>0$ depending only on $\lambda, \nu, M, U, c_{0}, n, p$ and $s$, but not on $r$

$$
u\left(x_{0}\right) \leq C r .
$$

Proof. Applying the result ii) of Lemma 2.2 for $\mu=1$, we get

$$
\max _{B\left(x_{0}, r / 2\right)} u \leq C\left(\min _{B\left(x_{0}, r / 2\right)} u+r\right)
$$

where $C$ is a positive constant depending only on $\lambda, \nu, M, U, n, p$ and $s$. Using Lemma 2.1, we obtain

$$
u\left(x_{0}\right) \leq \max _{B\left(x_{0}, r / 2\right)} u \leq C\left(\min _{B\left(x_{0}, r / 2\right)} u+r\right) \leq C(C r+r)=C_{1} r .
$$

Remark 2.1. If in (2.3) $L_{\text {loc }}^{p}(\Omega)$ is replaced by $L^{p}(\Omega)$, and if the constant $c_{0}$ in (2.4)-(2.6) does not depend on $s$, then clearly the constants in Lemmas 2.1-2.3 will not depend on $s$.

Proof of Theorem 2.1. Let $\Omega_{\varepsilon}=\{x \in \Omega / d(x, \partial \Omega)>\varepsilon\}$ for $\varepsilon \in\left(0, r_{0} / 4\right)$, where $r_{0}$ is as in Lemma 2.1. We shall prove that $\nabla u$ is bounded in $\Omega_{4 \varepsilon}$ by a constant depending only on $\lambda, \nu, M, n, p, U,\left|a_{i j}\right|_{0, \alpha, \Omega_{3 \varepsilon}},\left|b_{i}\right|_{0, \alpha, \Omega_{3 \varepsilon}}$, $\left|c_{i}\right|_{0, \Omega_{3 \varepsilon}},|d|_{0, \Omega_{3 \varepsilon}}$ and $\varepsilon$.

Let $x_{0} \in \Omega_{4 \varepsilon}$. We distinguish two cases:

i) $B\left(x_{0}, 2 \varepsilon\right) \subset[u>0]$

Applying Lemma 2.2 iii) with $\mu=2$ and $r=\varepsilon$, we get

$$
\sup _{B\left(x_{0}, \varepsilon\right)}|\nabla u| \leq C\left(\frac{\sup _{B\left(x_{0}, 2 \varepsilon\right)} u}{\varepsilon}+1\right) \leq C\left(\frac{U}{\varepsilon}+1\right) .
$$

where $C$ is a positive constant independent of $\varepsilon$.

ii) $B\left(x_{0}, 2 \varepsilon\right) \cap[u=0] \neq \emptyset$ 
Let $x \in B_{\varepsilon}\left(x_{0}\right)$ such that $u(x)>0$ and let $r(x)=\operatorname{dist}(x,[u=0])$ be the distance between $x$ and the set $[u=0]$. Clearly we have $B(x, r(x)) \subset[u>0]$. Moreover we claim that $r(x)<3 \varepsilon$ and $\overline{B(x, r(x))} \subset B\left(x_{0}, 4 \varepsilon\right)$.

Indeed we know that there exists $x^{\prime} \in B\left(x_{0}, 2 \varepsilon\right) \cap[u=0]$. So we have

$$
r(x) \leq\left|x-x^{\prime}\right| \leq\left|x-x_{0}\right|+\left|x_{0}-x^{\prime}\right|<\varepsilon+2 \varepsilon=3 \varepsilon .
$$

Now if $y \in \overline{B(x, r(x))}$, we have

$$
\left|x_{0}-y\right| \leq\left|x_{0}-x\right|+|x-y| \leq \varepsilon+r(x)<\varepsilon+3 \varepsilon=4 \varepsilon .
$$

Since $u>0$ in $B(x, r(x)), \overline{B(x, r(x))} \subset B\left(x_{0}, 4 \varepsilon\right), r(x)<4 \varepsilon<r_{0}$ and $\partial B(x, r(x)) \cap \partial[u>0] \neq \emptyset$, we deduce from Lemma 2.3 that $u(x) \leq C r(x)$ for some constant independent of $r(x)$. Applying Lemma 2.2 ii) and iii) with $\mu=1 / 2$ and $r=r(x)$, we get

$$
\sup _{B(x, r(x) / 4)}|\nabla u| \leq C\left(\frac{\sup _{B(x, r(x) / 2)} u}{r(x)}+1\right) \leq C
$$

where $C$ is a positive constant depending only on $\varepsilon$ and the data. In particular $|\nabla u(x)| \leq C$.

Since $\nabla u(x)=0$ a.e. in $B_{\varepsilon}\left(x_{0}\right) \cap[u=0]$, it follows that $\nabla u$ is uniformly bounded in $B\left(x_{0}, \varepsilon\right)$.

\section{BOUNDARY LiPSCHITZ CONTINUITY}

In this section we assume that $\varphi=0$ on a nonempty $C^{1,1}$ portion $T$ of $\Gamma_{1}$ and prove that $u$ is locally Lipschitz continuous up to $T$. In fact by the same arguments one can prove the same result for any $C^{1,1}$ portion of $\Gamma_{1}$ on which $\varphi$ is of class $C^{2}$. We need the following assumptions on the data.

$$
\begin{aligned}
& \varphi=0 \quad \text { on a nonempty } C^{1,1} \text { portion } T \text { of } \Gamma_{1} \\
& \forall x \in T \exists s>0: a, b_{i} \in C^{0,1}(\overline{B(x, s) \cap \Omega}), c_{i}, d \in L^{\infty}(B(x, s) \cap \Omega), \\
& i=1, \ldots, n \\
& c, f, \operatorname{div}(H) \in L^{p}(B(x, s) \cap \Omega), p>\frac{n}{1-\alpha}, \quad 0<\alpha<1 \\
& |H(x)| \leq c_{0} \quad \text { a.e. in } B(x, s) \cap \Omega \\
& c+f+\operatorname{div}(H) \leq c_{0} \quad \text { in } \mathcal{D}^{\prime}(B(x, s) \cap \Omega)
\end{aligned}
$$

where $c_{0}$ is a positive constant depending only on $s$.

The main result of this section is the following.

Theorem 3.1. Let $(u, \chi)$ be a solution of $(P)$. Then we have $u \in$ $C_{\mathrm{loc}}^{0,1}(\Omega \cup T)$. 
We shall first transform the problem locally. Indeed let $x_{0} \in T$. Then there exists a neighborhood $V$ of $x_{0}$ in $\mathbb{R}^{n}$ and a $C^{1,1}$ bijection $\Phi: Q \rightarrow V$ such that

$$
\Phi \in C^{1,1}(\bar{Q}), \Psi=\Phi^{-1} \in C^{1,1}(\bar{V}), \Phi\left(Q_{+}\right)=V \cap \Omega \text { and } \Phi\left(Q_{0}\right)=V \cap T,
$$

where

$$
\begin{aligned}
Q & =\left\{x=\left(x^{\prime}, x_{n}\right) \in \mathbb{R}^{n}:\left|x^{\prime}\right|<1 \text { and }\left|x_{n}\right|<1\right\} \\
Q_{+} & =Q \cap \mathbb{R}_{+}^{n}, \mathbb{R}_{+}^{n}=\mathbb{R}^{n} \cap\left[x_{n}>0\right] \\
Q_{0} & =Q \cap\left[x_{n}=0\right] .
\end{aligned}
$$

Now we set for $y \in Q_{+}$

$$
\begin{aligned}
v(y) & =u o \Phi(y), \quad \gamma(y)=\chi o \Phi(y) \\
b(y) & =|\operatorname{Jac} \Phi(y)|^{t} D \Psi(y) \operatorname{ao} \Phi(y) D \Psi(y) \\
\widetilde{G}(y) & =|\operatorname{Jac} \Phi(y)|^{t} D \Psi(y) G o \Phi(y) \\
\widetilde{g}(y) & =|\operatorname{Jac} \Phi(y)| g o \Phi(y) .
\end{aligned}
$$

Then one can verify that $(v, \gamma)$ satisfies

$$
(\widetilde{P})\left\{\begin{array}{l}
(v, \gamma) \in H^{1}\left(Q_{+}\right) \times L^{\infty}\left(Q_{+}\right) \text {such that: } \\
(i) \quad v \geq 0, \quad 0 \leq \gamma \leq 1, \quad v(1-\gamma)=0 \text { a.e. in } Q_{+} \\
(i i) v=0 \quad \text { on } Q_{0} \\
(i i i) \int_{Q_{+}}(b(x) \nabla v+v \widetilde{B}(x)+\gamma \widetilde{H}(y)) \cdot \nabla \zeta \\
\quad-(\gamma \widetilde{c}(y)+\widetilde{G}(y) . \nabla v+\widetilde{d}(y) v) \zeta d y=\int_{Q_{+}} \widetilde{f} \zeta d y \\
\quad \forall \zeta \in H_{0}^{1}\left(Q_{+}\right) .
\end{array}\right.
$$

It is then obvious that it suffices to prove Theorem 3.1 for a solution $v$ of $(\widetilde{P})$. Therefore we will perform the proof assuming that $\Omega=Q_{+}$and $\Gamma_{1}=Q_{0}$. The proof is based on the following lemma.

Lemma 3.1. Let $x_{0}=\left(x_{01}, \ldots, x_{0 n}\right) \in Q_{0}$ and $R>0$ such that $B^{+}\left(x_{0}, R\right)=B\left(x_{0}, R\right) \cap \mathbb{R}_{+}^{n} \subset Q^{+}$. Then we have for some positive constant $C$ depending only on $\lambda, \nu, n, M, U$ and $R$,

$$
u(x) \leq C\left|x-x_{0}\right| \quad \forall x \in B^{+}\left(x_{0}, R\right) .
$$

Proof. Let $x_{1}=x_{0}-R e_{n}$ and let $\Omega_{R}=B_{2 R}\left(x_{1}\right) \cap \Omega$. We consider the function $v(x)=\psi\left(d_{1}(x)\right)$ defined for $x \in \Omega_{R}$, where $d_{1}(x)=\left|x-x_{1}\right|-R$ and

$$
\psi(t)=-\frac{\beta}{\alpha} t+\frac{\beta}{\alpha^{2}} e^{2 \alpha R}\left(1-e^{-\alpha t}\right)
$$




$$
\begin{aligned}
& \alpha=\frac{(n M-\lambda)}{\lambda R}+\frac{K}{\lambda}+2 \nu, \\
& K=n \sqrt{n} \sup _{i, j \in\{1, \ldots, n\}, x \in \overline{\Omega_{R}}}\left|\frac{\partial a_{i j}}{\partial x_{i}}\right|, \quad \beta=\frac{c_{0}}{\lambda},
\end{aligned}
$$

and $c_{0}=c_{0}\left(x_{0}, R\right)$ is a constant from (3.5).

We claim that

$$
L v+c+f+\operatorname{div}(H) \leq \lambda\left(\psi^{\prime \prime}\left(d_{1}\right)+\alpha \psi^{\prime}\left(d_{1}\right)+\beta\right)=0 \quad \text { in } \mathcal{D}^{\prime}\left(\Omega_{R}\right) .
$$

Indeed we first have

$$
\begin{aligned}
\frac{\partial v}{\partial x_{i}} & =\psi^{\prime}\left(d_{1}\right) \frac{\partial d_{1}}{\partial x_{i}}=\psi^{\prime}\left(d_{1}\right) \frac{x_{i}-x_{1 i}}{\left|x-x_{1}\right|} \\
\frac{\partial^{2} v}{\partial x_{i} \partial x_{j}}= & \psi^{\prime \prime}\left(d_{1}\right) \frac{\partial d_{1}}{\partial x_{i}} \frac{\partial d_{1}}{\partial x_{j}}+\psi^{\prime}\left(d_{1}\right) \frac{\partial^{2} d_{1}}{\partial x_{i} \partial x_{j}} \\
= & \psi^{\prime \prime}\left(d_{1}\right) \frac{\left(x_{i}-x_{1 i}\right)\left(x_{j}-x_{1 j}\right)}{\left|x-x_{1}\right|^{2}} \\
& +\psi^{\prime}\left(d_{1}\right)\left(\frac{\delta_{i j}}{\left|x-x_{1}\right|}-\frac{\left(x_{i}-x_{1 i}\right)\left(x_{j}-x_{1 j}\right)}{\left|x-x_{1}\right|^{3}}\right) .
\end{aligned}
$$

Then we deduce from (3.7)-(3.8) that

$$
\begin{aligned}
& \operatorname{div}(a(x) \nabla v)=\sum_{i, j=1}^{n} a_{i j}(x) \frac{\partial^{2} v}{\partial x_{i} \partial x_{j}}+\frac{\partial a_{i j}}{\partial x_{i}} \frac{\partial v}{\partial x_{j}} \\
& =\frac{\psi^{\prime \prime}\left(d_{1}\right)}{\left|x-x_{1}\right|^{2}}\left(\sum_{i, j=1}^{n} a_{i j}(x)\left(x_{i}-x_{1 i}\right)\left(x_{j}-x_{1 j}\right)\right) \\
& +\frac{\psi^{\prime}\left(d_{1}\right)}{\left|x-x_{1}\right|}\left(\sum_{i, j=1}^{n} a_{i j} \delta_{i j}\right) \\
& -\frac{\psi^{\prime}\left(d_{1}\right)}{\left|x-x_{1}\right|^{3}}\left(\sum_{i, j=1}^{n} a_{i j}(x)\left(x_{i}-x_{1 i}\right)\left(x_{j}-x_{1 j}\right)\right) \\
& +\frac{\psi^{\prime}\left(d_{1}\right)}{\left|x-x_{1}\right|}\left(\sum_{i, j=1}^{n} \frac{\partial a_{i j}}{\partial x_{i}}\left(x_{j}-x_{1 j}\right)\right) \\
& \leq \lambda \psi^{\prime \prime}\left(d_{1}\right)+(n M-\lambda) \frac{\psi^{\prime}\left(d_{1}\right)}{\left|x-x_{1}\right|}+K \psi^{\prime}\left(d_{1}\right) \\
& \leq \lambda \psi^{\prime \prime}\left(d_{1}\right)+\left(\frac{(n M-\lambda)}{R}+K\right) \psi^{\prime}\left(d_{1}\right) .
\end{aligned}
$$


Moreover we have by (1.3)-(1.4) and (3.7)

$$
\begin{array}{r}
\operatorname{div}(v B(x))+C(x) \cdot \nabla v+d(x) v=(B(x)+C(x)) \cdot \nabla v+(d+\operatorname{div}(B)) v \\
\leq|B(x)+C(x)| \cdot|\nabla v| \leq 2 \lambda \nu \psi^{\prime}\left(d_{1}\right) .
\end{array}
$$

Using (3.5), (3.9) and (3.10), we get

$$
\begin{aligned}
L v+c+f+\operatorname{div}(H) & \leq \lambda \psi^{\prime \prime}\left(d_{1}\right)+\left(\frac{(n M-\lambda)}{R}+K+2 \lambda \nu\right) \psi^{\prime}\left(d_{1}\right)+c_{0} \\
& =\lambda\left(\psi^{\prime \prime}\left(d_{1}\right)+\alpha \psi^{\prime}\left(d_{1}\right)+\beta\right)=0
\end{aligned}
$$

which is (3.6).

Now we have

$$
u=0 \leq v \quad \text { on } \quad \overline{\Omega_{R}} \cap \Gamma_{1}
$$

and

$$
v=\psi\left(d_{1}(x)\right)=\psi(2 R-R)=\psi(R) \quad \text { on } \quad \Gamma_{R}=\partial \Omega_{R} \backslash \Gamma_{1} .
$$

If $\psi(R) \geq \max _{\overline{\Omega_{R}}} u$, then by (3.12), we have

$$
v \geq u \text { on } \Gamma_{R} .
$$

If $\psi(R)<\max _{\overline{\Omega_{R}}} u$, we take $v=k \psi\left(d_{1}\right)$, where $k=\max _{\overline{\Omega_{R}}} u / \psi(R)>1$.

Since $k \psi^{\prime \prime}\left(d_{1}\right)+\alpha k \psi^{\prime}\left(d_{1}\right)+\beta=\beta(1-k)<0, v$ satisfies (3.6) and also $v \geq u$ on $\partial \Omega_{R}$.

Now let $\zeta \in \mathcal{D}\left(\Omega_{R}\right), \zeta \geq 0$ and $\varepsilon>0$. Using (3.6) and $(P)$ iii) with the test function

$$
\xi=\min \left(\zeta, \frac{(u-v)^{+}}{\varepsilon}\right),
$$

we obtain by taking into account that $\chi=1$ a.e. in $[u>0]$

$$
\begin{aligned}
& \int_{\Omega_{R}}(a(x) \nabla u+u B(x)+H(x)) \cdot \nabla \xi-(c(x)+C(x) \cdot \nabla u+d(x) u) \xi d x \\
& =\int_{\Omega_{R}} f \xi d x \\
& -\int_{\Omega_{R}}(a(x) \nabla v+v B(x)+H(x)) \cdot \nabla \xi-(c(x)+C(x) \cdot \nabla v+d(x) v) \xi d x \\
& \leq-\int_{\Omega_{R}} f \xi d x .
\end{aligned}
$$

Adding (3.14) and (3.15), we obtain

$$
\begin{aligned}
& \int_{\Omega_{R}}(a(x) \nabla(u-v)+(u-v) B(x)) \cdot \nabla \xi \\
& -(C(x) \cdot \nabla(u-v)+d(x)(u-v)) \xi d x \leq 0
\end{aligned}
$$


which can be written

$$
\begin{aligned}
& \int_{\Omega_{R} \cap\left[\varepsilon \zeta \leq(u-v)^{+}\right]}\left(a(x) \nabla(u-v)^{+}+(u-v)^{+} B(x)\right) \cdot \nabla \zeta \\
& -\left(C(x) \cdot \nabla(u-v)^{+}+d(x)(u-v)^{+}\right) \zeta d x \\
& \leq-\frac{1}{\varepsilon} \int_{\Omega_{R} \cap\left[\varepsilon \zeta>(u-v)^{+}\right]}\left(a(x) \nabla(u-v)^{+}+(u-v)^{+} B(x)\right) \cdot \nabla(u-v)^{+} \\
& +\frac{1}{\varepsilon} \int_{\Omega_{R} \cap\left[\varepsilon \zeta>(u-v)^{+}\right]}\left(C(x) \cdot \nabla(u-v)^{+}+d(x)(u-v)^{+}\right)(u-v)^{+} d x \\
& \leq-\frac{1}{\varepsilon} \int_{\Omega_{R} \cap\left[\varepsilon \zeta>(u-v)^{+}\right]}(u-v)^{+}\left(B(x) \cdot \nabla(u-v)^{+}-C(x) \cdot \nabla(u-v)^{+}\right. \\
& \left.-d(x)(u-v)^{+}\right) d x \\
& \leq \int_{\Omega_{R} \cap\left[\varepsilon \zeta>(u-v)^{+}\right]} \zeta\left(|B(x)-C(x)| \cdot\left|\nabla(u-v)^{+}\right|\right. \\
& \left.+|d(x)|(u-v)^{+}\right) d x .
\end{aligned}
$$

Letting $\varepsilon \rightarrow 0$ in (3.16), we get $L(u-v)^{+} \geq 0$ in $\mathcal{D}^{\prime}\left(\Omega_{R}\right)$. Taking into account (3.11)-(3.13), we get (see [3, Theorem 8.1, page 179]) $(u-v)^{+} \leq 0$ in $\Omega_{R}$. This leads to $u \leq v$ in $\Omega_{R}$.

We deduce that for all $x \in \Omega_{R}$

$$
\begin{aligned}
u(x) & \leq v(x)=\left|v(x)-v\left(x_{0}\right)\right| \\
& \leq \sup _{0 \leq t \leq R} \psi^{\prime}(t)\left|x-x_{0}\right|=\psi^{\prime}(0)\left|x-x_{0}\right|=C(R)\left|x-x_{0}\right| .
\end{aligned}
$$

Since $B^{+}\left(x_{0}, R\right) \subset \Omega_{R}$, the lemma is proved.

Proof of Theorem 3.1. Let $x_{0} \in Q_{0}$ and $R>0$ such that $B^{+}\left(x_{0}, 3 R\right) \subset$ $\Omega$. We shall prove that $\nabla u$ is bounded in $B^{+}\left(x_{0}, R\right)$ by a constant $C$ depending only on $\lambda, \nu, M, U$ and $R$.

We distinguish two cases:

i) $B^{+}\left(x_{0}, 2 R\right) \subset[u>0]$

Since $u$ satisfies

$$
\left\{\begin{array}{l}
L(u)=c+f+\operatorname{div}(H) \quad \text { in } \quad B^{+}\left(x_{0}, 2 R\right) \\
0 \leq u \leq U \text { in } B^{+}\left(x_{0}, 2 R\right), \quad u=0 \quad \text { on } \quad B_{2 R}\left(x_{0}\right) \cap Q_{0}
\end{array}\right.
$$

we deduce that $u \in C^{1, \alpha}\left(B^{+}\left(x_{0}, 2 R\right) \cup\left(B_{2 R}\left(x_{0}\right) \cap Q_{0}\right)\right)$ (see [3, p. 212], Corollary 8.36 and the Remark after it). In particular we obtain $|\nabla u(x)| \leq$ $C$ for all $x \in B^{+}\left(x_{0}, R\right)$.

ii) $\exists x_{f} \in B^{+}\left(x_{0}, 2 R\right) \cap[u=0]$ 
Let $x \in B^{+}\left(x_{0}, R\right)$ such that $u(x)>0$ and let $r=\operatorname{dist}(x,[u=0])$ be the distance between $x$ and the set $[u=0]$. Remark that we have $r \leq\left|x-x_{f}\right|<$ $2 R$. Moreover $B_{r}(x) \cap \Omega \subset[u>0]$ and $\overline{B_{r}}(x) \cap \Omega \subset B_{3 R}^{+}\left(x_{0}\right) \cap \Omega$.

Indeed, if $y \in \overline{B_{r}}(x) \cap \Omega$, we have $\left|x_{0}-y\right| \leq\left|x_{0}-x\right|+|x-y|<R+r<$ $R+2 R=3 R$.

Again we distinguish two cases:

a) $\overline{B(x, r)} \cap Q_{0}=\emptyset$

In this case, we have by Lemma $2.3 u(x) \leq C r$, where $C$ depends only on $\lambda$, $\nu, M, U, n, p$ and $R$. Then by arguing exactly as in the proof of Theorem 2.1 , we can prove that $\nabla u$ is uniformly bounded in $B_{r / 2}(x)$ by a constant depending only on $\lambda, \nu, M, U, n, p$ and $R$.

b) $\exists y_{1} \in \overline{B(x, r)} \cap Q_{0}$

Again we have two cases:

a) $B(x, r / 4) \subset \Omega$

By Lemma 3.1, we have for all $y \in B_{r / 4}(x)$

$$
u(y) \leq C\left|y-y_{1}\right| \leq C\left(|y-x|+\left|x-y_{1}\right|\right) \leq C(r / 4+r)=\frac{5}{4} C r
$$

where $C$ depends only on $\lambda, \nu, M, U$ and $R$. Then by arguing exactly as in the proof of Theorem 2.1, we can prove that $\nabla u$ is uniformly bounded in $B_{r / 8}(x)$ by a constant depending only on $\lambda, \nu, n, p, M, U,\left|a_{i j}\right|_{0,1, B^{+}\left(x_{0}, 2 R\right)}$, $\left|b_{i}\right|_{0,1, B^{+}\left(x_{0}, 2 R\right)},\left|c_{i}\right|_{0, B^{+}\left(x_{0}, 2 R\right)},|d|_{0, B^{+}\left(x_{0}, 2 R\right)}$ and $R$.

в) $\exists y_{2} \in B(x, r / 4) \cap Q_{0}$

So we have $x \in B^{+}\left(y_{2}, r / 4\right)$. Note that $B^{+}\left(y_{2}, r / 2\right) \subset B^{+}\left(x_{0}, 3 R\right)$. Indeed for $y \in B^{+}\left(y_{2}, r / 2\right)$, one has

$$
\left|y-x_{0}\right| \leq\left|y-y_{2}\right|+\left|y_{2}-x\right|+\left|x-x_{0}\right| \leq r / 2+r / 4+R<2 R / 2+2 R / 4+R<3 R \text {. }
$$

Moreover by Lemma 3.1, we have

$$
u(y) \leq C\left|y-y_{2}\right| \leq \frac{C}{2} r \quad \forall y \in B^{+}\left(y_{2}, r / 2\right)
$$

where $C$ depends only on $\lambda, \nu, n, M, U$ and $R$.

We consider the function $u_{r}$ defined by

$$
u_{r}(z)=\frac{u\left(y_{2}+r z\right)}{r}, \quad z \in B^{+}(O, 1 / 2) .
$$

By (3.17), we see that $u_{r}$ is uniformly bounded in $B^{+}(O, 1 / 2)$, i.e. $u_{r}(z) \leq$ $C / 2 \forall z \in B^{+}(O, 1 / 2)$. Moreover, $u_{r}$ satisfies

$$
\left\{\begin{array}{c}
\operatorname{div}\left(a_{r}(z) \nabla u_{r}+u_{r} B_{r}(z)\right)-\left(r C_{r}(z) \nabla u_{r}+r^{2} d_{r} u_{r}\right)=r c_{r}+r f_{r}+\operatorname{div}\left(H_{r}\right) \\
\text { in } B^{+}(O, 1 / 2) \\
u_{r} \in C^{1, \alpha}\left(B(O, 1 / 2)^{+} \cup\left(B(O, 1 / 2) \cap Q_{0}\right)\right)(\text { see }[3, \text { p. 212]). }
\end{array}\right.
$$


Applying Corollary 8.36 of [3, p. 212] and taking into account the Remark after it, we get

$$
\begin{aligned}
\left|u_{r}\right|_{1, \alpha, \overline{B^{+}(O, 1 / 4)} \leq} & C\left(\left|u_{r}\right|_{0, B^{+}(O, 1 / 2)}+\left|r c_{r}\right|_{p, B^{+}(O, 1 / 2)}\right. \\
& \left.+\left|r f_{r}\right|_{p, B^{+}(O, 1 / 2)}+\left|\operatorname{div}\left(H_{r}\right)\right|_{p, B^{+}(O, 1 / 2)}\right),
\end{aligned}
$$

where $C$ depends only on dist $(\overline{B(O, 1 / 4)}, \partial B(O, 1 / 2)),\left|a_{i j}\right|_{0,1, B^{+}\left(X_{0}, 3 R\right)}$, $\left|b_{i}\right|_{0,1, B^{+}\left(X_{0}, 3 R\right)},\left|c_{i}\right|_{0, B^{+}\left(X_{0}, 3 R\right)},|d|_{0, B^{+}\left(X_{0}, 3 R\right)}, \lambda, \nu, M, U, n, p$, and $R$. In particular, $\left|\nabla u_{r}\right|_{0, \overline{B^{+}(O, 1 / 4)}}$ is uniformly bounded. Hence $|\nabla u(x)| \leq C$ since $x \in B^{+}\left(y_{2}, r / 4\right)$.

Finally because we have $\nabla u(x)=0$ a.e. in $B^{+}\left(x_{0}, R\right) \cap[u=0]$, it follows that $\nabla u$ is uniformly bounded in $B^{+}\left(x_{0}, R\right)$.

Acknowledgment. This work was initiated at KFUPM under Project No. FT/2005-10. It was completed at Fields Institute. I am grateful for these institutions for the excellent research facilities and financial support.

\section{References}

[1] Alt, H.W., Strömungen durch inhomogene poröse Medien mit freiem Rand. J. Reine Angew. Math. 305 (1979), 89-115.

[2] Challal, S., Lyaghfouri, A., On the continuity of the free boundary in problems of type $\operatorname{div}(a(x) \nabla u)=-(\chi(u) h(x))_{x_{1}}$, Nonlinear Anal. 62(2) (2005), 283-300.

[3] Gilbarg, D., Trudinger, N. S., Elliptic Partial Differential Equations of Second Order, Springer-Verlag, 1983.

[4] Lyaghfouri, A., The inhomogeneous dam problem with linear Darcy's law and Dirichlet boundary conditions, Math. Models Methods Appl. Sci. 8(6) (1996), 1051-1077.

AbDeslem Lyaghfouri

Fields Institute

222 College Street

Toronto M5T 3J1, CANada

E-MAIL: A.LYAGHFOURI@UTORONTO.CA

AND

King Fahd University of Petroleum and Minerals

P.O. Box 728

DHAHRAN 31261

SAUdi Arabia

E-MAIL: LYAGHFO@KFUPM.EDU.SA 